\title{
A Rigorous Analysis of Emotion of Human Being using an Event-B Approach
}

\author{
Nilima Salankar \\ Sir Padampat Singhania University, Udaipur
}

\author{
Prasun Chakrabarti \\ Sir Padampat Singhania University, Udaipur
}

\begin{abstract}
The paper entails a refinement approach using Event-B to develop model of emotion of human being. The proposed model indicates that when one type of emotion is active others are inactive and some factors are activated simultaneously which makes it difficult to judge the mental state of humans by simply observing single factor. The relationship among different types of emotions and analysis of emotion of human has been carried out using Pro-B model checker and animator.
\end{abstract}

\section{General Terms}

Machine, set theory,domain

\section{Keywords}

Event-B,emotion,refinement

\section{INTRODUCTION}

Event-B is a formal method that provides a complete framework for the development of the models. This technique consists of describing rigorously the problem in an abstract model, introducing solutions or design details in the refinement steps to obtain more concrete specifications. Psychological study using recording instruments has given scientists a great deal of information about the bodily events in emotion which are able to measure the heart rate, blood pressure, activity of the stomach and gastrointestinal system , level of harmones, breathing rate and depth and many other bodily conditions in emotion[1]. From literature we find that autonomic nervous system has two parts sympathetic and parasympathetic, in emotion the sympathetic system causes the discharge of the hormone epinephrine(adrenalin) and nonrepinephrine (noradrenalin). Epinephrine duplicates and strengthens many of the actions of the sympathetic system on various internal organs .It helps to mobilize sugar resources so that the muscles can use it rapidly. The major effect of norepinephrine is to constinctperipheral blood vessels and so to raise blood pressure. Other part of the autonomic nervous system called parasympathetic system tends to be active when we are calm and relaxed. In active aroused emotional states sympathetic activity predominates while in calmer state parasympathetic activity is dominant. Here we have specified a scheme for identification of human emotion using Event-B model. Event-B is a variant of B Method based on the technique of abstraction and refinement[2],[3].The important feature of this approach is to formally define an abstract model of a system independent of the architecture and successively refine it to a detailed design in a series of intermediate steps. Results obtainedusing Pro-B model checker[4],[5] to analyze invariant properties of the B models have been observed. In thiscontext an incremental development of a system to analyze human emotion has been proposed.

\section{An Event B System}

The notion of abstract machine and refinement is central to Event-B. An abstract machine consists of sets, constants and variable clauses modeled as set theoretic constants. The invariants are defined as predicates on state variables. In refinement steps guards may be strengthened, new events may be introduced and variables may be added or removed[6],[7],[8]. Abstract and concrete variables are related through gluing invariants. Event-B notation is based on set theory and most of it is self-explanatory. Some of the frequently used notations in our model are given in Table 1 and are explained to enhance the readability. Machine

$\mathrm{M}$

SETS

$\mathrm{S} 1, \mathrm{~S} 2, \mathrm{~S} 3 \ldots$

CONSTANTS

C

PROPERTIES

$\mathrm{P}$

VARIABLES

$\mathrm{v} 1, \mathrm{v} 2, \mathrm{v} 3 \ldots \ldots$

INVARIANTS I

INITIALIZATION

init

EVENTS

E1 =WHEN G1 THEN S1 END;

E2 =WHEN G2 THEN S2 END;

END

Figure 1. Event B Machine

Table 1. Relational Notations

\begin{tabular}{|l|l|}
\hline Notations & Meanings \\
\hline & Mapping \\
\hline $\operatorname{dom}(\mathrm{R})$ & Domain of relation R \\
\hline $\operatorname{ran}(\mathrm{R})$ & Range of Relation R \\
\hline $\mathrm{R}$ & Domain restriction \\
\hline $\mathrm{R}[\mathrm{A}]$ & $\begin{array}{l}\text { Relational image of R over } \\
\text { set A }\end{array}$ \\
\hline
\end{tabular}


Let $A$ and $B$ be two sets, then relational constructor(1) defines the set of relations between $\mathrm{A}$ and $\mathrm{B}$ as:

$A \perp B=P(A \times B)$ where $x$ is Cartesian product of $A$ and $B$. A mapping of element $a: A$ and $b: B$ in relation $R \quad A \perp B$ is written as $a \mathrm{~m} b$. The domain of a relation $R: A \perp B$ is the set of elements of $A$ that $R$ relates to some elements in $B$ defined as:

$$
\operatorname{dom}(R)=\{a \mid a: A \& \# b .(b: B \& a m b: R)\}
$$

Similarly, the range of relation $R: A 1 B$ is defined as set of elements in $B$ related to some element in $A$ defined as :

$$
\operatorname{ran}(R)=\{b \mid b: B \& \# a .(a: A \& a m b: R)\}
$$

A relation $R: A \perp B$ can be projected on a domain $U$ (A called domain restriction $(R)$ defined as:

$$
U R R=\{a m b \mid a m b: R \& a: U\}
$$

The Relational image $R[U]$ where $U$ ( $A$ is defined as:

$$
R[U]=\{b \mid \# a . a m b: R \& a: U\}
$$

The Relational inverse $\left(R^{-1}\right)$ of a relation $R$ is defined as:

$$
R^{-1}=\{b m a \mid a m b: R)
$$

A function is a relation with Cartesian restrictions[9],[10]. The function may be partial function(2) or a total function (3). A partial function from set $A$ to $B(A 2 B)$ is a relation which relates an element in $A$ to at most one element in $B$. A total function from set $A$ to $B(A 3 B)$ is a partial function where $\operatorname{dom}(f)=A$, i.e. each element of set $A$ is related to exactly one element of set $B$. In the guarded statement, the guard $(G)$ of the event is expressed as a first order predicate. The action of events are specified as simultaneous assignments of state variables usingsubstitution statements[11],[12]. Events occur spontaneously whenever their guards hold true and they are executed automatically[13]. Structure of machine is given in Figure 1.

\section{Model}

The proposed model consists of $p$ samples and $c$ conditions resulted as a emotion where $p>2$ and $\mathrm{c} \geq 2$, where $\mathrm{c} 1 \neq \mathrm{c} 2$ and $\mathrm{p} 1 \neq \mathrm{p} 2$. The system is asynchronous such that no bound can be placed on the time required on diagnosis of type. It is assumed that diagnosis faults may occur. Formally a system described in terms of six elements.

$\mathrm{P}=\{\mathrm{p} 1, \mathrm{p} 2, \ldots, \mathrm{pn}\} \quad$ No of samples

$\mathrm{C}=\{\mathrm{c} 1, \mathrm{c} 2, . ., \mathrm{cn}\} \quad$ Types of emotion

$\mathrm{T}=\{\mathrm{t} 1, \mathrm{t} 2, . ., \mathrm{tn}\} \quad$ Set of time values

$\mathrm{Y}=\{\mathrm{y} 1, \mathrm{y} 2, \ldots, \mathrm{yn}\} \quad$ Set of outputs

$X=\{x 1, x 2, \ldots, x n\} \quad$ Set of time dependent input functions

$\mathrm{Y}=\{\mathrm{y} 1, \mathrm{y} 2, \ldots, \mathrm{yn}\} \quad$ Set of time dependent output functions

Intensity(fear, anger) $\alpha$ Sympathetic System

Intensity(relax, calm) $\alpha$ Parasympathetic System

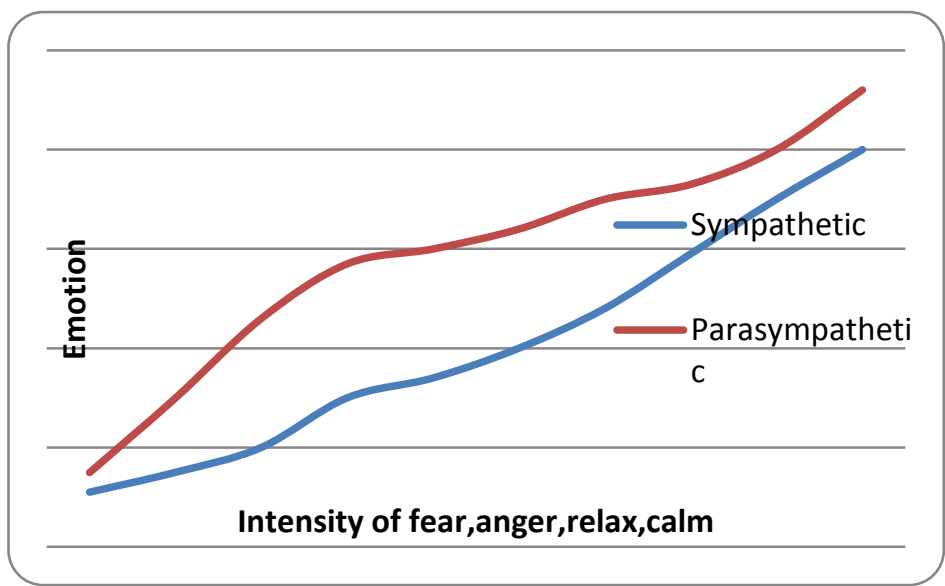

Figure 2. The intensity of the emotion directly proportional to fear, anger, relax and calm

\section{Flow of model}

To analyze the type of emotion stated in section proposed model, we have used an approach of construction of abstract model and further introduced refinement of abstract model by introducing more variables and operations as shown in figure 3 .In refinementwe have introduced variables fear, anger, DDOTTY

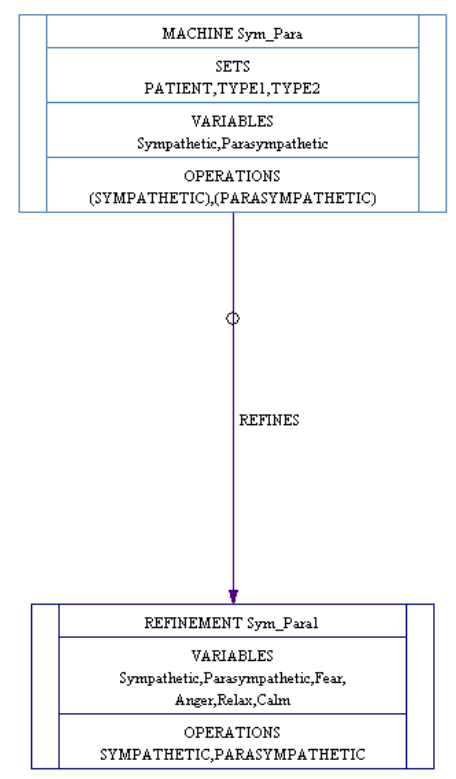

Figure 3. Flow of Model

\section{Flow of Model}

In this section development of a system of emotion has been outlined as below:

\section{Outline of the refinement steps}

In this development begin with abstract model of emotions and successively refine it to a next model which categorize the 
sympathetic and parasympathetic based on specific types of emotion. Incremental development of the system consists of following steps.

a. Abstract Model consists of a division of a autonomic nervous system. We have assumed that division is mutually exclusive. Once the diagnosis falls into the category of Type1 then it no longer available for the another diagnosis of Type2.

b. Refinement outlines how fear, anger, relax and calm state of mind helps us to determine exact state of mind.

\section{Abstract Model of emotion}

*Sympathetic and Parasympathetic autonomic nervous system*/

MACHINE Sym_Para

SETS

$P A T I E N T=\{p 1, p 2, p 3, p 4, p 5, p 6, p 7\} ; T Y P E 1=\{s y\} ; T Y P E 2=\{p a\}$

VARIABLES Sympathetic,Parasympathetic

INVARIANT

/*I1*/Sympathetic:PATIENT+->TYPE1 \&

/*I2*/ Parasympathetic:PATIENT +->TYPE2

INITIALIZATION

Sympathetic: $=\{\}||$ Parasympathetic: $=\{\}$

OPERATIONS

$\operatorname{SYMPATHETIC}(p p, t t)=$

PRE pp:PATIENT\& $t t: T Y P E 1$ THEN

SELECT pp/:dom(Sympathetic)

\&pp/:dom(Parasympathetic) THEN

Sympathetic: $=$ Sympathetic $\bigvee\{p p \mid->t t\}$

END

END;

PARASYMPATHETIC $(p p, t t)=$

PRE pp:PATIENT\& $t t: T Y P E 2$ THEN

SELECT pp/:dom(Parasympathetic)

\&pp/:dom(Sympathetic) THEN

Parasympathetic: $=$ Parasympathetic $\vee\{p p \mid->t t\}$

END

END

END
The abstract model of a emotion MACHINE Sym_Para is given as B machine developed on Pro-B is given in Figure 4. The PATIENT,TYPE1 and TYPE2 sets define types for model. The variable sympathetic is defined as partial function from PATIENT to TYPEI in invariant I-1. It contains mapping from PATIENT toTYPE1. It indicates that patient $\mathrm{pp}$ has been diagnosed for type tt. The partial function ensures that type is associated with only one patient. The variable parasympathetic is a relation between PATIENT and TYPE2 defined in invariant $I-2$. This mapping indicates that a patient $\mathrm{pp}$ has been diagnosed for type tt. The sympathetic and parasympathetic variables are initialized as empty sets. In this machine a diagnosed patients are added to the particular set. It may be noticed that all diagnosed patients must be removed from to be diagnose list.

\section{Introducing fear, anger, relax and calm}

/*Sympathetic(Fear \& Anger) and Parasympathetic(Relax and Calm) Situation of Stress*/

\section{REFINEMENT Sym_Paral}

REFINES Sym_Para

VARIABLES

Sympathetic,Parasympathetic,Fear,Anger,Relax,Calm

\section{INVARIANT}

/*I3*/Fear :PATIENT+->TYPE1 \&

/*I4*/Anger:PATIENT+->TYPE1 \&

/*I5*/Relax:PATIENT+->TYPE2 \&

/*I6*/Calm :PATIENT+->TYPE2

\section{INITIALIZATION}

Sympathetic: $=\{\}||$ Parasympathetic: $:=\{\} \|$ Fear $:=\{\}||$ Anger $:=\{\} \|$ Relax $:=\{\} \|$ Calm: $:=\{\}$

\section{OPERATIONS}

\section{$\operatorname{SYMPATHETIC}(p p, t t)=$}

PRE pp:PATIENT\& tt:TYPE1 THEN

SELECT pp/:dom(Fear) \&pp/:dom(Anger) THEN

Sympathetic: $=$ Sympathetic $\bigvee\{p p \mid->t t\} \|$

Fear: $=$ Fear $\vee\{p p \mid->t t\} \|$

Anger: $=$ Anger $\bigvee\{p p \mid->t t\}$

END

END;

$\operatorname{PARASYMPATHETIC}(p p, t t)=$

PRE pp:PATIENT\& $t t: T Y P E 2$ THEN

SELECT pp/:dom(Relax) \&pp/:dom(Calm) $\& p p /: \operatorname{dom}($ Fear) \&pp/:dom(Anger) THEN 


\author{
Parasympathetic: $=$ Parasympathetic $\bigvee\{p p \mid->t t\}||$ \\ Relax: $=\operatorname{Relax} \bigvee\{p p \mid->t t\} \|$ \\ Calm: $=\operatorname{Calm} \bigvee\{p p \mid->t t\}$
}

END

END

$E N D$

Figure 5. Introducing fear, anger, relax and calm

The refinement of abstract model of emotion REFINEMENT Sym_Paral is given in Figure 5, various others variables are introduced. The invariant corresponding to fear, anger,relax and calm are given in I-3,I-4,I-5andI-6.

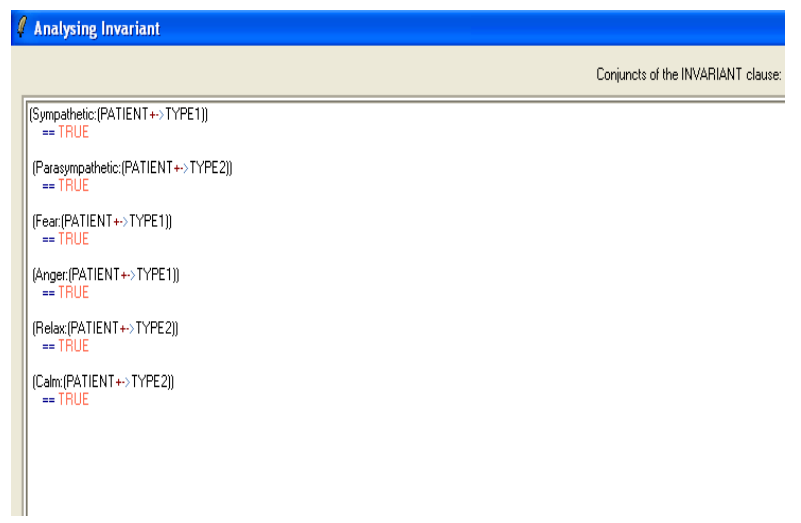

Figure 6. Invariant Status During Model Check

After developing the model of emotion, the objective is to verify whether the proposed model preserves the properties of emotion and maintains the mutual exclusiveness on the basis of identified factors. The Pro-B tool supports automatic consistency checking of B machines via model checking. However for exhaustive model checking given sets of machine must be restricted to small finite sets and integer variables must be limited to small numeric ranges. After addition of all invariants,model has beenchecked, and all invariants which have designed for analyzing properties of emotion are true for random animation as given in Figure 6.The state of a model after random animation is given in Figure 7.

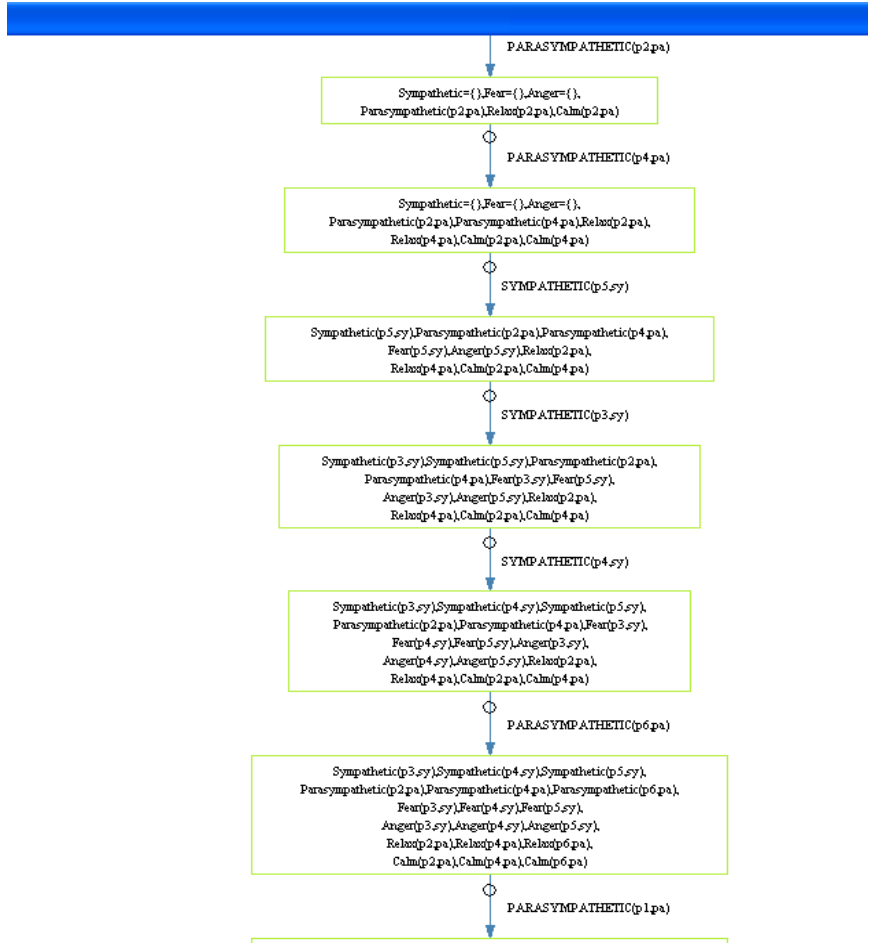

Figure 7. State of a model after random animation

\section{CONCLUSION}

In this paper an incremental construction of a model of human emotion has been proposed and analysis of the B specifications and invariant properties of this system using Pro-B Model Checker and Animator has been carried out.The experimental results strengthen the fact that abstraction and refinement are valuable techniques for development of models. The Event-B model of emotion also reveals that exhaustive model checking of the specifications on B Model checker provides a clear insight into the system and develops the understanding towards realization of the satisfiabilityof invariant properties.

\section{REFERENCES}

[1] Morgan C,KingR,WeiszJ,Schopler J. Introduction to Psychology,Tata McGraw-Hill Edition,New Delhi,2011,307-316.

[2] C Metayer, J R Abrial, and L Voison. Event-B language.RODIN deliverables 3.2, http:// rodin.cs.ncl.ac.uk/deliverables/ D7.pdf, 2005.

[3] Defago, Schiper, and Urban: Total order broadcast and multicast algorithms: Taxonomy and survey. ACM Computing.Surveys. 36(4), 372-421,2004

[4] Michael Leuschel, Michael Butler, ProB: A Model Checker for B, FME 2003, Volume 2805 of LNCS, 855874, Springer,2003.

[5] J.-R. Abrial.The B-Book: Assigning programs to meanings. Cambridge University Press, 1996

[6] N.Fulmare, D.Yadav, Rigorous analysis of byzantine causal order using Event-B,ISBN:978-1-60558-812doi>10.1145/1741906.1742074 
[7] P. M. Melliar-Smith, Louise E. Moser, and VivekAgrawala. Broadcast protocols for distributed systems. IEEE Trans. Parallel Distributed Systems, 1(1):17-25, 1990.

[8] Kenneth P. Birman, Andre Schiper, and Pat Stephenson. Lightweigt causal and atomic group multicast. $A C M$ Trans. Comput. Syst., 9(3):272-314, 1991

[9] DivakarYadav and Michael Butler. Rigorous design of fault-tolerant transactions for replicated database systems using Event B, Volume 4157 of LNCS, 343-363, Springer, 2006.

[10] DivakarYadav and Michael Butler, Formal Development of Broadcast Systems and Verification of Ordering
Properties using Event-B, Volume 5454 of LNCS, 152176, Springer ,2009.

[11] Fekete, A., Kaashoek, M.F., Lynch, N.: Implementing sequentially consistent shared objects using broadcast and point-to-point communication. Journal of the ACM, 45(1), 35-69, 1998.

[12] Fekete, A., Lynch, N.A., Shvartsman, A.A.: Specifying and using a partitionable group communication service. ACM Trans. Comput. Syst. 19(2), 171-216,2001

[13]Louise E. Moser and P.M.Melliar-Smith, Byzantine Resistant Total Ordering Algorithms, Information and Computation 150, 75-111,1999. 